\title{
Scriptaid affects histone acetylation and the expression of development-related genes at different stages of porcine somatic cell nuclear transfer embryo during early development
}

\author{
ZHOU Yan ${ }^{1 \dagger}$, HUANG YongYe ${ }^{1 \dagger}, X$ X WanHua ${ }^{1}$, SONG Qi ${ }^{1}$, JI Yuan $^{1}$, ZHANG YanPing $^{2}$, \\ OUYANG HongSheng ${ }^{1}$, LAI LiangXue ${ }^{1}$, PANG DaXin ${ }^{1} \&$ TANG XiaoChun ${ }^{1 *}$ \\ ${ }^{1}$ Jilin Provincial Key Laboratory of Animal Embryo Engineering, College of Animal Science and Veterinary Medicine, Jilin University, \\ Changchun 130062, China; \\ ${ }^{2}$ College of Life Science and Technology, Dalian University, Dalian 116622, China
}

Received September 23, 2012; accepted January 8, 2013; published online May 6, 2013

\begin{abstract}
Although the somatic cell nuclear transfer (SCNT) technique has been used extensively for cloning and generating transgenic pigs, the cloning efficiency is still very low. It has been proposed that the low efficiency of this technique is the result of incomplete epigenetic reprogramming and abnormal gene expression during early embryonic development. In this study, we investigate the effect of Scriptaid, a low-toxicity histone deacetylase inhibitor, on the developmental competence of porcine SCNT embryos. We found that treating SCNT embryos with $500 \mathrm{nmol} / \mathrm{L}$ Scriptaid for $15 \mathrm{~h}$ after activation significantly enhanced the blastocyst formation rate $(27.7 \%)$ compared with the untreated group (control) $(12.2 \%, P<0.05)$. Using an immunofluorescence technique to measure the average fluorescence intensity, we also found that treating SCNT embryos with Scriptaid increased the level of histone acetylation on histone $\mathrm{H} 3$ at lysine 14 (acH3K14). Furthermore, treating embryos with Scriptaid increased the expression level of three genes that play important roles during embryonic development (Oct4, Klf4 at the blastocyst stage and Nanog at the 4-cell stage). Moreover, the expression level of the apoptosis-related gene Caspase-3 was significantly lower in the Scriptaid-treated SCNT embryos compared with the control SCNT embryos at the 4-cell and blastocyst stages. In conclusion, these results indicate that Scriptaid treatment improves the development and nuclear reprogramming of porcine SCNT embryos.
\end{abstract}

porcine, Scriptaid, epigenetic reprogramming, histone acetylation, development-related genes

Citation: Zhou Y, Huang Y Y, Xie W H, et al. Scriptaid affects histone acetylation and the expression of development-related genes at different stages of porcine somatic cell nuclear transfer embryo during early development. Chin Sci Bull, 2013, 58: 2044-2052, doi: 10.1007/s11434-013-5827-x

In 2000, the birth of the world's first cloned pig using somatic cell nuclear transfer (SCNT) created a new range of applications for somatic cell-cloned pigs in biomedical research $[1,2]$. Because of its similarity to humans in terms of breeding characteristics and physiology, the pig is considered an excellent donor for xenotransplantation and has been extensively used in human disease models [3]. A low cloning efficiency $(<1 \%)$, however, has restricted the extensive use of porcine SCNT in biomedical research, and it has been proposed that this low efficiency is primarily caused

$\dagger$ These authors contributed equally to this work.

*Corresponding author (email: xiaochuntang@jlu.edu.cn) by inadequate epigenetic reprogramming [4]. Previous studies have shown that the incomplete reprogramming of SCNT embryos results in abnormal gene expression patterns [5]. As a consequence of these abnormal expression patterns, the cloned embryos may stop developing and be aborted [6].

Epigenetic modifications made to the genome include genomic methylation and histone modifications [7,8]. Histone acetylation can alter chromosome conformation, which can increase gene transcription and translation [9]. Histone deacetylase inhibitor (HDACi) can increase the acetylation level of core histones in cloned bovine embryos [10]. This finding demonstrates that HDACi induces hyperacetylation and the expression of key genes that are important for 
SCNT embryonic development.

Currently, several types of HDACi have been used successfully to enhance the nuclear reprogramming of SCNT embryos. Valproic acid, for example, can significantly improve the epigenetic reprogramming of SCNT embryos in vitro $[11,12]$. After Trichostatin A (TSA) treatment, the developmental ability of pre-implantation SCNT embryos is enhanced in several species, such as mice [13], cattle [14] and pigs [15]. These findings demonstrate that TSA can improve cloning efficiency, but TSA treatments at higher concentrations or over longer durations can have harmful effects on SCNT embryonic development in vitro and may cause developmental defects $[16,17]$. Scriptaid is a synthetic HDACi that induces histone acetylation and chromosome remodeling and enhances gene transcription [18]. A previous study has shown that the development of cloned inbred mouse embryos improved significantly in vitro and in vivo after Scriptaid treatment and that nascent mRNA expression levels were higher in the treated group compared with the control groups [19]. Furthermore, treating porcine SCNT embryos with Scriptaid also improved their in vitro developmental capability and their nuclear reprogramming $[16,20]$. Similar results were also obtained in bovine embryos [21].

Previous studies have shown that Scriptaid has a positive effect on the in vitro and in vivo development of porcine embryos, but its underlying mechanism remains to be elucidated. For instance, the effect of Scriptaid treatment on gene expression remains unknown. In the present study, we aimed to determine the optimal concentration of Scriptaid for treatment. We also investigated the relationship among histone acetylation (acH3K14), pluripotent gene expression (Oct4, Sox2, Klf4 and Nanog), apoptosis-related gene expression (Bcl-xl, Caspase-3 and Bak) and Scriptaid treatment at different early developmental stages in porcine SCNT embryos. We investigate whether treating embryos with Scriptaid can change the expression levels of these genes and make them similar to the expression levels observed in the conventional in vitro fertilized (IVF) embryos.

\section{Materials and methods}

All of the chemicals used in this study were purchased from the Sigma Chemical Company (St Louis, MO, USA) unless otherwise noted. All of the solutions and media mentioned were filtered through a $0.22-\mu \mathrm{m}$ filter. All of the animal experiments were approved by the Animal Care and Use Committee of Jilin University and performed in accordance with the Animal Welfare and Ethics Guidelines.

\subsection{Establishment of donor cell}

All of the pigs used in this study were from the Jilin University swine herd. Landrace fetal fibroblast cells (FFCs) were used for SCNT and were prepared as previously described [22]. First, fetuses were collected from pregnant sows at approximately day 35 . The head, viscera and limbs were removed, and the remaining fetal tissues were cut into very fine pieces $\left(1 \mathrm{~mm}^{3}\right)$ with ophthalmic scissors. The tissue pieces were digested with Collagenase IV $(200 \mathrm{U} / \mathrm{mL})$ and DNase I (25 U/mL) in DMEM containing $20 \%$ fetal bovine serum for $4-6 \mathrm{~h}$ at $39^{\circ} \mathrm{C}$ with $5 \% \mathrm{CO}_{2}$ in the atmosphere. Following the digestion, the cell pellet was pooled in a $10-\mathrm{cm}$ culture dish and allowed to propagate until confluent approximately $12 \mathrm{~h}$. The cells were cultured for several passages. The cells were frozen and used between passages 2 and 6 for SCNT. The cultured cells were separated into single cells using a trypsin digestion and used as donor cells $0.5 \mathrm{~h}$ before performing SCNT.

\subsection{Preparation of SCNT embryos}

For the preparation of SCNT embryos, a slightly modified version of a previously described protocol was used [22]. Pig ovaries were obtained from local slaughter houses and transported to the laboratory in sterile $0.9 \% \mathrm{NaCl}$ solution with antibiotics at $32-37^{\circ} \mathrm{C}$ within $4 \mathrm{~h}$. Follicles with a diameter of 3-6 mm were selected, and the cumulus oocyte complexes (COCs) were collected by aspirating with a syringe. The COCs with more than three layers of intact cumulus cells were cultured in a $35-\mathrm{mm}$ dish and allowed to mature for $42-44 \mathrm{~h}$ at $39^{\circ} \mathrm{C}$ in a $5 \% \mathrm{CO}_{2}$ atmosphere. After maturation, the cumulus cells were removed from the COCs in $1 \%$ PVA-TL-Hepes containing $0.1 \%$ hyaluronidase by pipetting repeatedly. The matured oocytes with protruding first polar body (PB) and a substantial cytoplasm were selected for the production of SCNT or IVF embryos. Metaphase II (MII) oocytes were transferred into manipulation medium (25 mmol/L Hepes-buffered TCM-199 with 3 $\mathrm{mg} / \mathrm{mL}$ BSA) with $0.75 \mathrm{mg} / \mathrm{mL}$ cytochalasin B. The PB along with a small amount of surrounding cytoplasm, was aspirated using a beveled glass pipette to enucleate the oocyte. After enucleation, the donor cell was transferred by injecting a single fetal fibroblast cell into the perivitelline space closest to cytoplasm of the recipient oocyte. Finally, groups of 20 reconstructed complexes were fused and activated with 2 direct current pulses ( $1 \mathrm{~s}$ interval) of $1.2 \mathrm{kV} / \mathrm{cm}$ for $30 \mu$ s using a BTX ECM 2001 Electro Cell Manipulator (BTX, San Diego, CA, USA) in fusion groove. The successfully reconstructed embryos were kept in Porcine Zygote Medium-3 (PZM3, pH 7.3) supplemented with $3 \mathrm{mg} / \mathrm{mL} \mathrm{BSA}$ at $39^{\circ} \mathrm{C}$ and in a $5 \% \mathrm{CO}_{2}$ atmosphere. The 2and 4-cell cleavage rates and the blastocyst formation rate were recorded at 24,48 and $144 \mathrm{~h}$, respectively, with the activation time designated as $0 \mathrm{~h}$.

\subsection{In vitro fertilization}

For the preparation of IVF embryos, a slightly modified 
version of a previously described protocol was used [23]. Mature oocytes without cumulus cells were washed three times in mTBM medium containing $1 \mathrm{mmol} / \mathrm{L}$ caffeine. Groups of 40 oocytes were cultured in $100 \mu \mathrm{L}$ drops of mTBM medium and equilibrated for $30 \mathrm{~min}$. The spermatozoa were washed three times in PBS with $0.1 \%$ BSA by centrifugation at $900 \times g$ for $5 \mathrm{~min}$, and then re-suspended in mTBM medium. The sperm samples were added to fertilization drops containing the oocytes for a final sperm concentration of $5 \times 10^{5}$ cells $/ \mathrm{mL}$, and the oocytes were cocultured with the spermatozoa in mTBM for $5 \mathrm{~h}$. Finally, the zygotes were washed three times, and groups of $50 \mathrm{zy}-$ gotes were cultured in $100 \mu \mathrm{L}$ of PZM3 medium drops at $39^{\circ} \mathrm{C}$ in a $5 \% \mathrm{CO}_{2}$ atmosphere.

\subsection{Scriptaid treatment}

Scriptaid was dissolved in dimethyl sulfoxide and prepared as a $1000 \times$ stock solution at $500 \mu \mathrm{mol} / \mathrm{L}$. The stock solution was stored under dark conditions and added to PZM3 culture media at different concentrations using serial gradient dilutions. Any remaining stock solutions were stored at $4{ }^{\circ} \mathrm{C}$ for no more than 2 weeks. To determine the optimal concentration for treatment, the porcine embryos were treated with $0,300,400,500,600,700,800$ and $1000 \mathrm{nmol} / \mathrm{L}$ Scriptaid for $15 \mathrm{~h}$ after activation. To determine the optimal treatment duration, the porcine embryos were cultured with $500 \mathrm{nmol} / \mathrm{L}$ Scriptaid for $0,8,15,24$ and $48 \mathrm{~h}$ after activation. After Scriptaid treatment, the culture medium was changed to PZM3 without Scriptaid.

\subsection{Detection of acH3K14 and Oct4 using immunofluorescence}

Histone acetylation and Oct4 expression levels were determined using indirect immunofluorescence. The Scriptaid-treated and non-treated embryos were collected at the 2-cell (24 h), 4-cell (48 h) and blastocyst (144 h) stages. Embryos were fixed for $30 \mathrm{~min}$ in $4 \%$ (w/v) paraformaldehyde, permeabilized for 30 min with $0.2 \%$ Triton X-100 in PBS, and then blocked in 5\% goat serum in PBS for $30 \mathrm{~min}$ at room temperature. Next, the embryos were incubated in a rabbit polyclonal antibody to histone $\mathrm{H} 3$ acetyl K14 (Abcam, Cambridge, UK) diluted 1:50 or a rabbit polyclonal antibody to Oct-3/4 (H-134) (Santa Cruz Biotechnology, CA, USA) diluted 1:200 overnight at $4^{\circ} \mathrm{C}$. After washing extensively with $0.2 \%$ Tween-20 in PBS, the embryos were then incubated in an Alexa Fluor-594-labeled goat antirabbit IgG (Invitrogen, Carlsbad, CA, USA) diluted 1:800 for $1 \mathrm{~h}$ at $39^{\circ} \mathrm{C}$. The embryos were mounted on slides in mounting medium containing $10 \mu \mathrm{g} / \mathrm{mL}$ Hoechst 33342 to stain DNA for 2 min. Images were captured using a fluorescent microscope (Nikon, Japan) and the appropriate excitation wavelength and exposure times. The images of the embryos were analyzed using the Image Pro Plus 6.0 soft- ware (Media Cybernetics, USA). The average ratios of the acH3K14 and Oct4 signals were calculated and compared among the different groups. To diminish any errors in the fluorescence measurements, each experiment was repeated at least three times with 10-20 embryos in each replication.

\subsection{Preparation of single-embryo cDNA and real-time PCR}

The single cell RNA-Seq method has been previously described in detail [24]. In this study, we used a slightly modified version of this method to prepare single-embryo cDNA. Briefly, the zona pellucida was removed at the 2-, 4-cell and blastocysts stages from Scritptaid treated, untreated and IVF embryos by incubating the embryos in acidic Tyrode's solution for $1 \mathrm{~min}$. First-strand cDNA was synthesized using SuperScript III reverse transcriptase (Invitrogen) and UP1 primer. The second strand was amplified using TaKaRa Ex Taq $^{\mathrm{TM}}$ HS DNA Polymerase (TaKaRa Bio, Japan) and UP2 Primer. The cDNA was amplified by 20 cycles of PCR. For each group, we analyzed three embryos that were randomly selected from the same batch at each stage. The cDNA from the embryos was diluted ten-fold in nuclease-free water and used as the template for real-time PCR. Real-time PCR was performed using the BioEasy SYBR Green I Real Time PCR Kit (Bioer, Hangzhou, China) on the $\mathrm{iQ}^{\mathrm{TM}} 5$ system (Bio-Rad, USA). All PCR reactions were performed in triplicate. All of the PCR amplification was performed using the following protocol: $94^{\circ} \mathrm{C}$ for $10 \mathrm{~s}$, then $54 / 60^{\circ} \mathrm{C}$ for $15 \mathrm{~s}$, and extension at $72^{\circ} \mathrm{C}$ for $30 \mathrm{~s}$ for 40 cycles. The sequences of the primers for each assayed gene are shown in Table 1.

\subsection{Counting nuclei of blastocysts and statistical analysis}

Blastocysts with good morphology at day 6 were selected and fixed in $4 \%$ paraformaldehyde in PBS for $30 \mathrm{~min}$ at room temperature. The embryos were mounted on slides in mounting medium containing $10 \mu \mathrm{g} / \mathrm{mL}$ of Hoechst 33342 . The number of cells in each blastocyst was determined using a fluorescent microscope with UV light. All of the embryos were collected at random from each treatment group, and each analysis was conducted at least three times. The cleavage rate data were analyzed using the Chi-Square test. Other data were analyzed by one-way ANOVA with SPSS 16.0 (SPSS Inc., Chicago, IL, USA). $P<0.05$ was considered to be statistically significantly different.

\section{Results}

\subsection{Scriptaid effect on the development of SCNT embryos in vitro}

After activation, SCNT embryos were treated with Scriptaid at a concentration of 0 (control), 300, 400, 500, 600, 700, 
Table 1 Real-time PCR primers and PCR conditions

\begin{tabular}{|c|c|c|c|c|}
\hline Gene & Primer sequences $\left(5^{\prime} \rightarrow 3^{\prime}\right)$ & Annealing temperature $\left({ }^{\circ} \mathrm{C}\right)$ & Product length (bp) & Accession number \\
\hline Oct4 & $\begin{array}{l}\text { F: AAGCAGTGACTATTCGCAAC } \\
\text { R: CAGGGTGGTGAAGTGAGG }\end{array}$ & 60 & 136 & NM_001113060.1 \\
\hline Sox 2 & $\begin{array}{l}\text { F: CGCAGACCTACATGAACG } \\
\text { R: TCGGACTTGACCACTGAG }\end{array}$ & 60 & 103 & NM_001123197.1 \\
\hline Klf4 & $\begin{array}{l}\text { F: GTTCTCATCTCAAGGCACACC } \\
\text { R: TCGCACTTCTGGCACTGG }\end{array}$ & 60 & 157 & NM_001031782.1 \\
\hline Nanog & $\begin{array}{l}\text { F: TCCTCTTCCTTCCTCCAT } \\
\text { R: TCCTTCTCTGTGCTCTTC }\end{array}$ & 60 & 108 & NM_001129971.1 \\
\hline$\beta$-actin & $\begin{array}{l}\text { F: GAACCCCAAAGCCAACCGT } \\
\text { R: CCTCGTAGATGGGCACCGT }\end{array}$ & 60 & 173 & U07786.1 \\
\hline$B c l-x l$ & $\begin{array}{l}\text { F: TTTTCTCCTTCGGTGGGG } \\
\text { R: GCATTGTTTCCGTAGAGTTCC }\end{array}$ & 54 & 169 & NM_214285.1 \\
\hline Caspase-3 & $\begin{array}{l}\text { F: GAAGACCATAGCAAAAGGAGCA } \\
\text { R: TTTGGGTTTGCCAGTTAGAGTT }\end{array}$ & 54 & 156 & NM_214131.1 \\
\hline$B a k$ & $\begin{array}{l}\text { F: TACCTTACCCTGGGAGTGGC } \\
\text { R: GGAAAACCTCCTCCGTGTC }\end{array}$ & 54 & 205 & XM_001928147.2 \\
\hline GAPDH & $\begin{array}{l}\text { F: GCCATCACCATCTTCCAGG } \\
\text { R: TCACGCCCATCACAAACAT }\end{array}$ & 54 & 190 & NM_001206359.1 \\
\hline
\end{tabular}

800 and $1000 \mathrm{nmol} / \mathrm{L}$ in PZM3 for $15 \mathrm{~h}$. The 2- and 4-cell cleavage rates and the blastocyst formation rate were determined at 24, 48 and $144 \mathrm{~h}$, respectively, and are shown in Table 2. The blastocyst formation rate significantly increased when SCNT embryos were treated with 300, 400, 500 and $600 \mathrm{nmol} / \mathrm{L}$ Scriptaid compared with the control group $(20.7 \%, 22.1 \%, 27.7 \%, 23.5 \%$ vs. $12.2 \%, P<0.05)$. The total cell number of the blastocysts and their cleavage rate when treated with different concentrations of Scriptaid were not significantly different compared with the nontreated embryos. Based on these results, all of the following experiments were conducted using $500 \mathrm{nmol} / \mathrm{L}$ Scriptaid for $15 \mathrm{~h}$ after activation.

To better investigate the effects of Scriptaid, we analyzed the in vitro development of SCNT embryos treated with 500 nmol/L Scriptaid for $0,8,15,24$ and $48 \mathrm{~h}$. As shown in Table 3 , the blastocyst formation rate of SCNT embryos treated with $500 \mathrm{nmol} / \mathrm{L}$ Scriptaid for $15 \mathrm{~h}(28.1 \%)$ was higher than the rates for embryos treated for 8,24 or $48 \mathrm{~h}$ and for the non-treated embryos $(19.6 \%, 17.5 \%, 18.7 \%$ and $14.0 \%)$. Increasing the treatment duration, however, slightly decreased the 2- and 4-cell cleavage rates and blastocyst formation rate of SCNT embryos. The different Scriptaid treatment duration did not have any significant effect on the average number of cells within the blastocysts.

\subsection{Scriptaid effect on histone acetylation}

To determine the level of the histone acetylation in SCNT embryos treated with $500 \mathrm{nmol} / \mathrm{L}$ Scriptaid for $15 \mathrm{~h}$, the intensity of the epigenetic marker acH3K14 was measured at the 2-cell, 4-cell and blastocyst stages of Scriptaid-treated and control SCNT embryos. As shown in Figure 1 , the average optical intensity of the fluorescence signal for acH3K14 increased in SCNT embryos treated with Scriptaid at 2-cell (0.048 vs. 0.029), 4-cell (0.055 vs. 0.043), and blastocyst stages ( 0.033 vs. 0.023$)$, compared with control embryos.

Table 2 Effects of different Scriptaid concentration on the development of SCNT Embryos in vitro ${ }^{\text {a) }}$

\begin{tabular}{|c|c|c|c|c|c|c|}
\hline $\begin{array}{l}\text { Concentration of } \\
\text { Scriptaid (nmol/L) }\end{array}$ & $\begin{array}{c}\text { No. of } \\
\text { embryos }\end{array}$ & Replications & $\begin{array}{c}\text { No. of cleaved embryos (\%) } \\
\text { at } 24 \mathrm{~h}\end{array}$ & $\begin{array}{c}\text { No. of cleaved embryos }(\%) \\
\text { at } 48 \mathrm{~h}\end{array}$ & $\begin{array}{c}\text { No. of blastocysts }(\%) \\
\text { at } 144 \mathrm{~h}\end{array}$ & $\begin{array}{l}\text { Total cell No. in } \\
\text { blastocyst }\end{array}$ \\
\hline 0 (control) & 98 & 4 & $58(59.2)$ & 78 (79.6) & $12(12.2)^{\mathrm{a}}$ & $39.5 \pm 6.8^{\mathrm{a}}$ \\
\hline 300 & 116 & 4 & $71(61.2)$ & $103(88.7)$ & $24(20.7)^{\mathrm{b}}$ & $35.4 \pm 4.5^{\mathrm{a}}$ \\
\hline 400 & 95 & 4 & $58(60.1)$ & $77(81.1)$ & $21(22.1)^{b}$ & $36.2 \pm 3.1^{\mathrm{a}}$ \\
\hline 500 & 90 & 4 & $58(64.4)$ & $77(85.5)$ & $25(27.7)^{b}$ & $37.6 \pm 4.9^{\mathrm{a}}$ \\
\hline 600 & 89 & 4 & $60(67.4)$ & $73(82.0)$ & $21(23.5)^{b}$ & $39.2 \pm 4.1^{\mathrm{a}}$ \\
\hline 700 & 131 & 4 & $78(59.5)$ & 103 (78.6) & $25(19.1)^{\mathrm{ab}}$ & $35.8 \pm 2.6^{\mathrm{a}}$ \\
\hline 800 & 71 & 4 & $42(59.1)$ & $58(81.7)$ & $13(18.3)^{\mathrm{ab}}$ & $34.2 \pm 2.3^{\mathrm{a}}$ \\
\hline 1000 & 118 & 4 & $68(57.6)$ & $92(77.9)$ & $17(14.4)^{\mathrm{a}}$ & $35.4 \pm 4.5^{\mathrm{a}}$ \\
\hline
\end{tabular}

a) a, b values with different superscripts within each column are significantly different $(P<0.05)$; cell number in blastocyst: mean \pm SD. 
Table 3 Effects of different $500 \mathrm{nmol} / \mathrm{L}$ Scriptaid treatment durations on the development of SCNT Embryos in vitro ${ }^{\text {a) }}$

\begin{tabular}{|c|c|c|c|c|c|c|}
\hline $\begin{array}{l}\text { Duration of } \\
\text { treatment }(\mathrm{h})\end{array}$ & $\begin{array}{l}\text { No. of } \\
\text { embryos }\end{array}$ & Replications & $\begin{array}{c}\text { No. of cleaved embryos (\%) } \\
\text { at } 24 \mathrm{~h}\end{array}$ & $\begin{array}{c}\text { No. of cleaved embryos (\%) } \\
\text { at } 48 \mathrm{~h}\end{array}$ & $\begin{array}{c}\text { No. of blastocysts (\%) } \\
\text { at } 144 \mathrm{~h}\end{array}$ & $\begin{array}{c}\text { Total cell No. in } \\
\text { blastocyst }\end{array}$ \\
\hline 0 & 100 & 4 & $54(54.0)$ & $75(75.0)$ & $14(14.0)^{\mathrm{a}}$ & $36.2 \pm 2.4^{\mathrm{a}}$ \\
\hline 8 & 158 & 3 & $88(55.7)$ & $131(82.9)$ & $31(19.6)^{b}$ & $38.3 \pm 6.4^{\mathrm{a}}$ \\
\hline 15 & 110 & 4 & $67(60.1)$ & $86(78.1)$ & $31(28.1)^{b}$ & $37.8 \pm 4.1^{\mathrm{a}}$ \\
\hline 24 & 107 & 4 & $63(58.5)$ & $87(81.3)$ & $19(17.5)^{\mathrm{ab}}$ & $37.2 \pm 5.4^{\mathrm{a}}$ \\
\hline 48 & 80 & 4 & $42(52.5)$ & $61(76.2)$ & $15(18.7)^{\mathrm{ab}}$ & $36.4 \pm 4.9^{\mathrm{a}}$ \\
\hline
\end{tabular}

a) a, b values with different superscripts within each column are significantly different $(P<0.05)$; cell number in blastocyst: mean \pm SD.
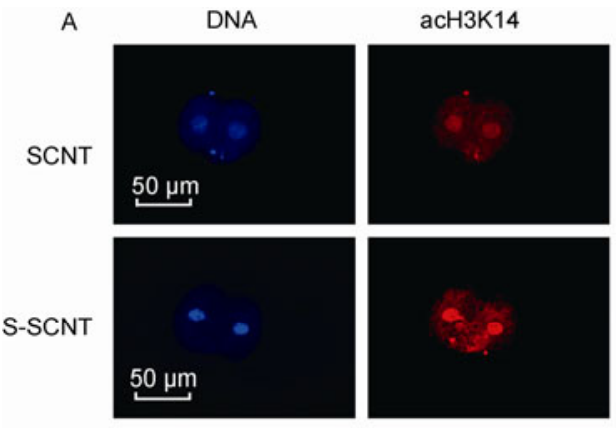

B

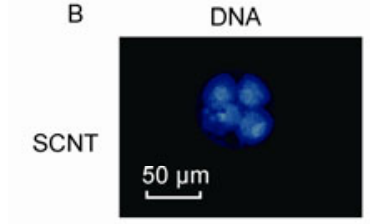

acH3K14
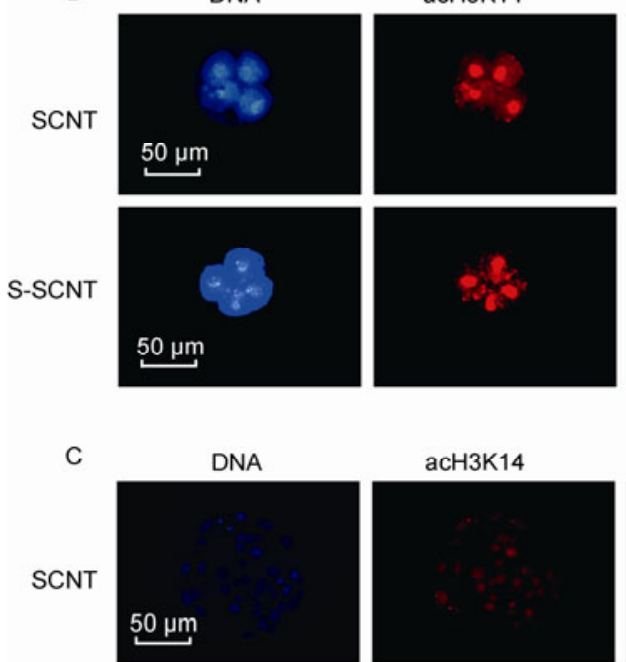

S-SCNT

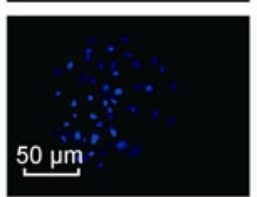

acH $3 \mathrm{~K} 14$
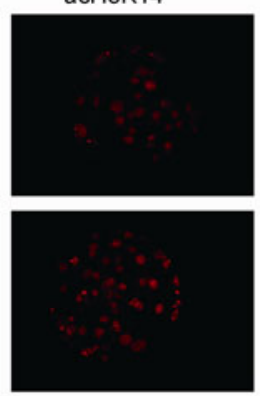

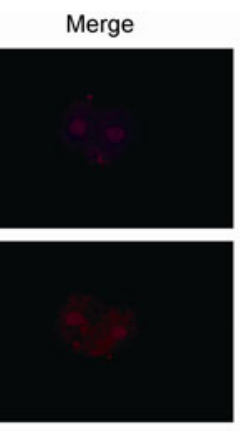

Merge
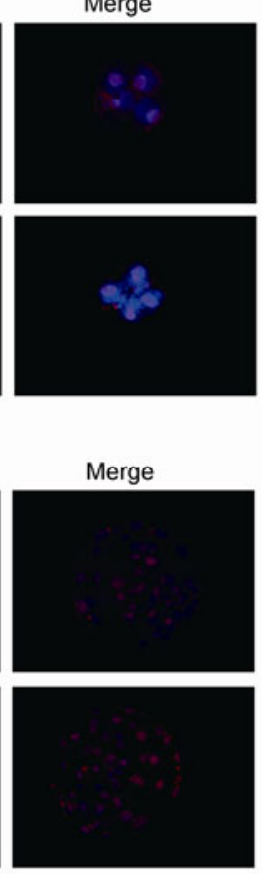
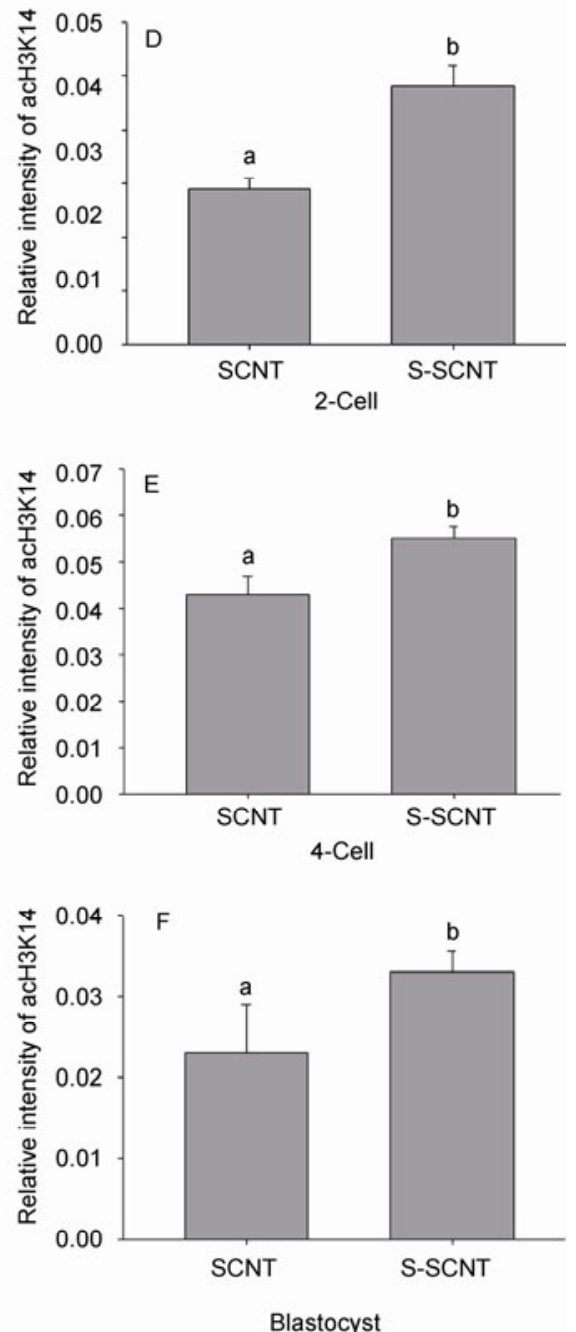

Figure 1 Histone acetylation level, as measured by acH3K14 staining, in SCNT and S-SCNT embryos at the 2-cell (A, D), 4-cell (B, E) and blastocyst (C, F) stages. The embryos were labeled with acH3K14 (red) and with Hoechst 33342 for DNA (blue). SCNT, non-treated embryos; S-SCNT, Scriptaid-treated embryos. $n=15-20$. a, b superscripts denote significant differences between SCNT and S-SCNT $(P<0.05)$.

\subsection{Scriptaid effect on the expression of pluripotency- related genes and apoptosis-related genes}

To investigate the effects of Scriptaid treatment on gene expression patterns during early embryonic development, the expression levels of four pluripotency-related genes (Oct4, Sox2, Klf4 and Nanog) and three apoptosis-related genes (Bcl-xl, Caspase-3 and Bak) were determined in Scriptaid-treated SCNT embryos, non-treated SCNT embryos, and IVF embryos at the 2-, 4-cell and blastocyst stages.

As shown in Figure 2, the expression levels of the four pluripotency-related genes steadily increased from the 2-cell to blastocyst stage. The expression levels of Oct4, Sox 2 and 

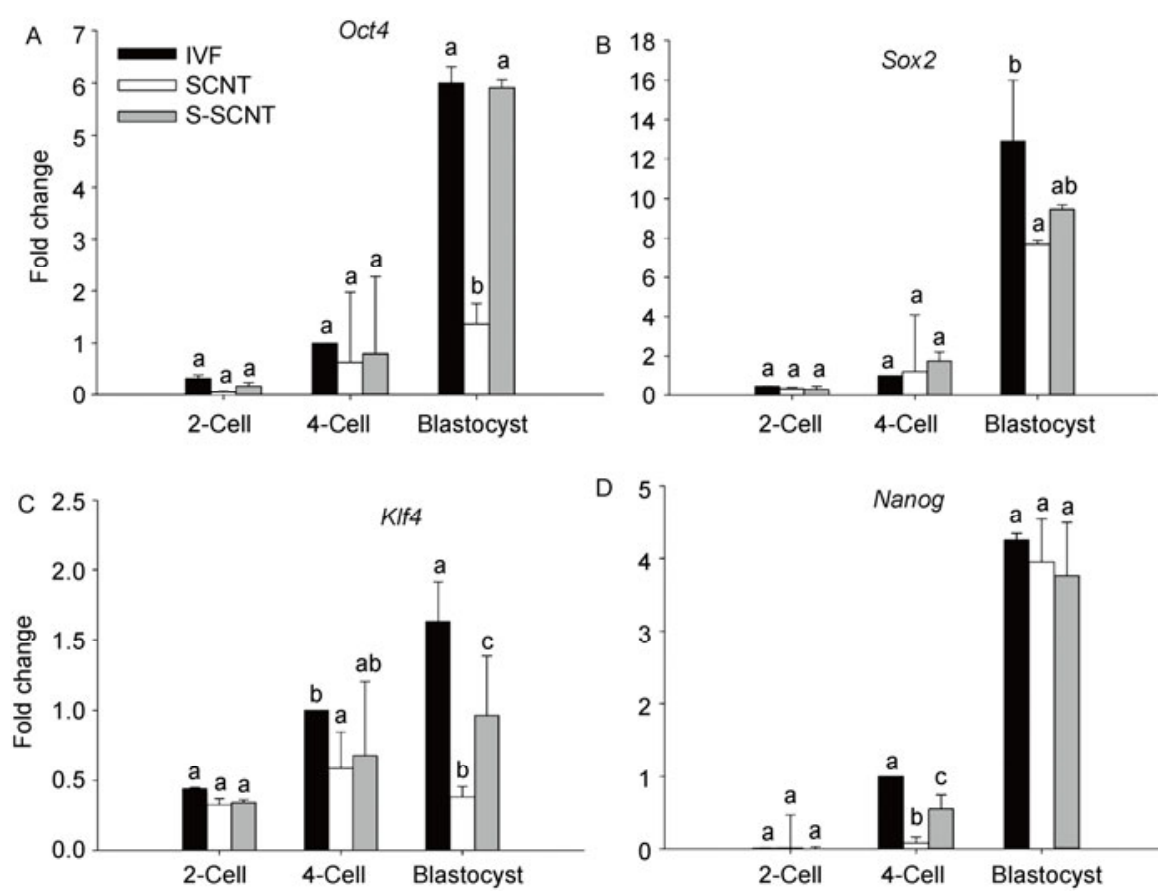

Figure 2 The relative expression patterns of $O c t 4$ (A), Sox2 (B), Klf4 (C) and Nanog (D) at the 2-cell, 4-cell and blastocyst stages in IVF, SCNT, and S-SCNT embryos during pre-implantation development. IVF, in vitro fertilization; SCNT, non-treated embryos; S-SCNT, Scriptaid-treated embryos. a, b, c values with different superscripts are significantly different $(P<0.05)$.

Klf4 in the SCNT blastocysts were lower than the expression levels observed in the IVF embryos. The expression levels of Oct4 and Klf4 were higher in the Scriptaid-treated group at the blastocyst stage compared with the non-treated groups (no significant difference in Sox2 expression). Notably, the expression level of Oct4 at the blastocyst stage in the Scriptaid-treated group was similar to the expression level observed in the IVF embryos. The expression level of

Nanog at the 2-cell stage was barely detectable. At the blastocyst stage, the expression level of Nanog was similar in the Scriptaid-treated and non-treated groups, but at the 4-cell stage, the expression level of Nanog was significantly higher in the Scriptaid-treated SCNT embryos compared with the untreated embryos.

Next, we further investigated the gene expression pattern changes that were observed in the SCNT embryos treated with Scriptaid. Using real-time PCR, our study shows that Scriptaid treatment can restore the Oct4 expression level to the level observed in IVF embryos at the blastocyst stage. Therefore, we therefore further investigated the Oct4 expression pattern at the blastocyst stage using immunofluorescence, and the results are shown in Figure 3. The protein expression level of Oct4 at the blastocyst stage was higher in the Scriptaid-treated embryos compared with the untreated groups $(0.053$ vs. $0.039, P<0.05)$.

We also determined the expression levels of apoptosis-related genes (Bcl-xl, Caspase-3 and Bak) at different developmental stages. As shown in Figure 4, the expression level of $B c l-x l$ gradually decreased from the 2-cell to
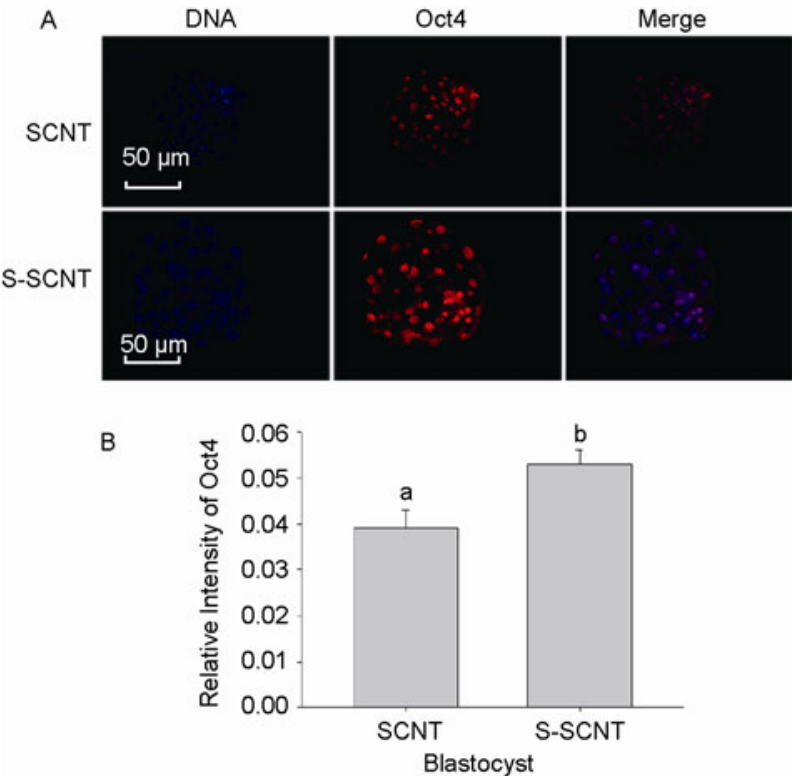

Figure 3 The expression of Oct4 at the blastocyst stage in SCNT and $\mathrm{S}$-SCNT embryos determined by indirect immunofluorescence. (A) Images of SCNT and S-SCNT embryos at the blastocyst stage. (B) Comparison of Oct4 expression between SCNT and S-SCNT embryos. SCNT, non-treated embryos; S-SCNT, Scriptaid-treated embryos. $n=15-20$. a, b superscripts denote significant differences between SCNT and S-SCNT embryos $(P<0.05)$.

blastocyst stage, and the expression levels of Caspase-3 and $B a k$ increased from the 2-cell to blastocyst stage. The expression level of $B c l-x l$ in the SCNT group was lower than 

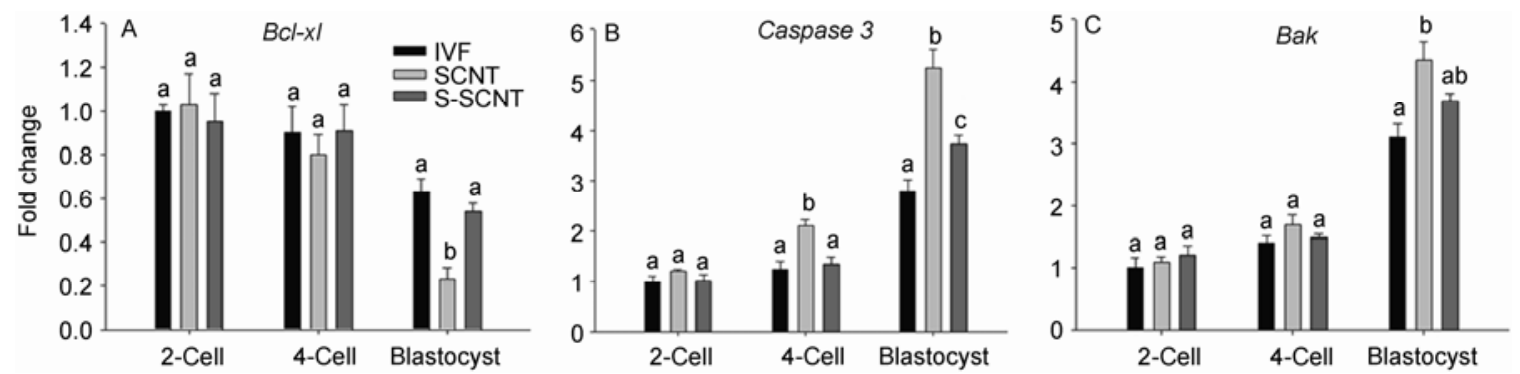

Figure 4 The relative expression patterns of Bcl-xl (A), Caspase-3 (B) and Bak (C) at the 2-cell, 4-cell and blastocyst stages in IVF, SCNT, and S-SCNT embryos during pre-implantation development. IVF, in vitro fertilization; SCNT, non-treated embryos; S-SCNT, Scriptaid-treated embryos. a, b values with different superscripts are significantly different $(P<0.05)$

the expression level in the IVF and Scriptaid-treated group at blastocyst stage. The expression level of Caspase-3 in the SCNT group was higher than the expression level in the IVF and Scriptaid-treated group at the 4-cell and blastocyst stages. Additionally, the expression level of Caspase-3 in the Scriptaid-treated embryos was higher than the expression level in the IVF group. We also found that the expression level of Bak was similar between the Scriptaid treated and untreated SCNT groups.

\section{Discussion}

Recently, a growing body of evidence had indicated that the low success rate for cloning and the developmental defects found in cloned embryos can be attributed to incomplete epigenetic reprogramming, which includes abnormal epigenetic modifications, such as DNA methylation and histone modifications, in SCNT embryos [25]. Therefore, we used HDAC inhibitors to improve the epigenetic modifications and increase the developmental competence of SCNT embryos.

In this study, we investigated the effects of Scriptaid on the in vitro developmental potential of SCNT embryos. When treated with Sciptaid for a longer time period (24, $48 \mathrm{~h}$ ), we found that the blastocyst formation rate of the embryos was higher than that of the non-treated group. We found no significant difference in the 2- and 4-cell cleavage rates between these groups. Furthermore, when a higher concentration $(700,800 \mathrm{nmol} / \mathrm{L})$ of Scriptaid was used, the blastocyst rate was even higher compared to that of the control group. These findings show that Scriptaid has a lower toxicity level in SCNT embryo and are consistent with those of previous studies in mice [19]. Furthermore, the in vitro developmental capability of SCNT embryos treated with Scriptaid improved significantly compared with that of the control group. The blastocyst rate was approximately 2-fold higher in the Scriptaid-treated group than in the control group (27.7\% vs. $12.2 \%)$.

Regarding the mechanism underlying the Scriptaid effect on the reprogramming of SCNT embryos, histone acetylation-deacetylation is a dynamic process during embryonic development, and increasing histone acetylation relaxes the chromosome structure. This relaxed structure may induce a transcriptionally permissive state [26,27], which may facilitate successful cloning [28]. The key genes for embryonic development are more likely to be activated after histone hyperacetylation. Studies have shown that treating embryos with Scriptaid inhibits histone deacetylase and results in hyperacetylation, which can expose DNA active binding sites and decrease DNA methylation that are important for gene activation [29]. Therefore, gene activation in embryos could transform the transcriptional quiescence genome into a transcriptional activated state [30].

H3K14 is an important site for histone acetylation [31]. In this study, we found that the average intensity of acetylation on H3K14 in SCNT embryos increased at the 2-, 4-cell and blastocyst stages when the embryos were treated with Scriptaid. This result indicates that the level of histone acetylation in the SCNT embryos treated with Scriptaid increased when compared to that in the control group. These results are consistent with previous findings for other histone acetylation sites, including H4K5 and H3K9 [21].

To determine whether Scriptaid can activate the expression of pivotal genes through histone acetylation, we examined the relative mRNA expression levels of four genes that play important roles during development, Oct4, Sox 2 , Klf4 and Nanog. Oct4 plays a critical role in establishing the three cell lineages and in mammalian pre-implantation embryonic development [32]. It is expressed at a significantly lower level in SCNT blastocysts compared with IVF blastocysts [33]. Furthermore, Oct4 is initially activated in embryos during the pre-implantation period and can form a heterodimer with Sox2, which results in these two proteins binding DNA together [34]. Klf4 can activate Nanog through the p53 pathway and improve the production of induced pluripotent cells. Klf4 is considered a good indicator of pluripotency, and deleting the expression of Klf4 in mouse embryos results in early death [35]. Sox2 and Nanog are essential transcription factors that maintain the selfrenewal of undifferentiated embryonic stem cells and establish embryonic identity. More importantly, previous studies have shown that co-expressing Sox2, Oct4 and Nanog can form an automatic regulatory network that can establish the 
porcine embryonic developmental potential [36]. We found that treating embryos with Scriptaid significantly increased the transcriptional activity of Oct4 and Klf4 at the blastocyst stage and Nanog at the 4-cell stage compared with the control group. Furthermore, the level of Oct4 expression in the Scriptaid-treated embryos is similar to the expression level observed in IVF embryos. These results indicate that Scriptaid can increase the transcriptional activity of genes in SCNT embryos and affirm the importance of Oct4, Klf4 and Nanog expression during reprogramming. Furthermore, this finding also indicates that embryonic gene activation may occur at the 4-cell stage in pig embryos. It should be noted that the Oct4 expression level in SCNT embryos treated with a histone deacetylase inhibitor treatment varies among the different studies that have been reported. In bovine SCNT embryos treated with Oxamflatin, the Oct4 expression level was significantly higher than in the IVF group at the blastocyst stage [37]. Similar to the finding from the present study, porcine embryos treated with TSA appeared to have a similar Oct4 expression level compared with IVF embryos at the blastocyst stage [38]. The different Oct4 expression levels observed in embryos treated with a histone deacetylase inhibitor may be result of different treatment compounds and differing culture condition.

Apoptosis occurs frequently early embryonic development and has a significant impact on the development of the embryo [39]. Therefore, we investigated the expression patterns of three apoptosis-related genes, Bcl-xl, Bak and Caspase-3. Bcl-xl, which inhibits apoptosis, and Caspase-3 and $B a k$, which promote apoptosis, belong to the $B c l-2$ family. Currently, at least 11 caspase genes have been identified that mediate protein cleavage and induce apoptosis. Among them, Caspase- 3 has been found to execute apoptosis. In this study, we found that the expression levels of Caspase-3 and Bak gradually increased from the 2-cell to the blastocyst stage of embryonic development. After treating embryos with Scriptaid, the expression level of Caspase-3 was lower than in the non-treated embryos at the 4-cell and blastocyst stages. This result indicates that Scriptaid treatment enhances the vitality of the blastocyst. The expression level of the apoptosis-inhibiting gene $B c l-x l$, however, also decreased during early embryonic development. We cannot exclude the possibility that the in vitro culture environment gradually deteriorated during the culture progress.

In conclusion, our study shows that Scriptaid can improve the blastocyst formation rate of SCNT embryos in vitro and can change the expression patterns of specific genes that are important in early embryonic development. These finding suggest that Scriptaid is beneficial for the nuclear reprogramming of early porcine SCNT embryos and may be a useful reagent for the efficient cloning pig embryos. Future studies should investigate whether Scriptaid affects the expression of other genes, and determine what additional steps are required to improve the remodeling and reprogramming of pig embryos. Furthermore, many reports have indicated that the use of histone deacetylase inhibitors has adverse effects [40-42]. Therefore, further studies should also determine whether Scriptaid can be safely used during embryo development and whether it has any adverse effects.

We thank everyone involved for their assistance with this project. This work was supported by the National Basic Research Program of China (2009CB941000).

1 Polejaeva I A, Chen S H, Vaught T D, et al. Cloned pigs produced by nuclear transfer from adult somatic cells. Nature, 2000, 407: 86-90

2 Onishi A, Iwamoto M, Akita T, et al. Pig cloning by microinjection of fetal fibroblast nuclei. Science, 2000, 289: 1188-1190

3 Lai L, Kolber-Simonds D, Park K W, et al. Production of alpha-1,3galactosyltransferase knockout pigs by nuclear transfer cloning. Science, 2002, 295: 1089-1092

4 Whitworth K M, Prather R S. Somatic cell nuclear transfer efficiency: How can it be improved through nuclear remodeling and reprogramming? Mol Reprod Dev, 2010, 77: 1001-1015

5 Cezar G G. Epigenetic reprogramming of cloned animals. Cloning Stem Cells, 2003, 5: 165-180

6 Jeanisch R, Eggan K, Humpherys D, et al. Nuclear cloning, stem cells, and genomic reprogramming. Cloning Stem Cells, 2002, 4: 389-396

7 Bird A. DNA methylation patterns and epigenetic memory. Genes Dev, 2002, 16: 6-21

8 Li E. Chromatin modification and epigenetic reprogramming in mammalian development. Nat Rev Genet, 2002, 3: 662-673

9 Struhl K. Histone acetylation and transcriptional regulatory mechanisms. Genes Dev, 1998, 12: 599-606

10 Oliveira C S, Saraiva N Z, de Souza M M, et al. Effects of histone hyperacetylation on the preimplantation development of male and female bovine embryos. Reprod Fertil Dev, 2010, 22: 1041-1048

11 Miyoshi K, Mori H, Mizobe Y, et al. Valproic acid enhances in vitro development and Oct-3/4 expression of miniature pig somatic cell nuclear transfer embryos. Cell Reprogram, 2010, 12: 67-74

12 Huang Y, Tang X, Xie W, et al. Histone deacetylase inhibitor significantly improved the cloning efficiency of porcine somatic cell nuclear transfer embryos. Cell Reprogram, 2011, 13: 513-520

13 Kishigami S, Mizutani E, Ohta $\mathrm{H}$, et al. Significant improvement of mouse cloning technique by treatment with trichostatin A after somatic nuclear transfer. Biochem Biophys Res Commun, 2006, 340: 183-189

14 Akagi S, Matsukawa K, Mizutani E, et al. Treatment with a histone deacetylase inhibitor after nuclear transfer improves the preimplantation development of cloned bovine embryos. J Reprod Dev, 2011, 57: 120-126

15 Yamanaka K, Sugimura S, Wakai T, et al. Acetylation level of histone $\mathrm{H} 3$ in early embryonic stages affects subsequent development of miniature pig somatic cell nuclear transfer embryos. J Reprod Dev, 2009, 55: 638-644

16 Zhao J, Ross J W, Hao Y, et al. Significant improvement in cloning efficiency of an inbred miniature pig by histone deacetylase inhibitor treatment after somatic cell nuclear transfer. Biol Reprod, 2009, 81: 525-530

$17 \mathrm{Wu} \mathrm{X,} \mathrm{Li} \mathrm{Y,} \mathrm{Li} \mathrm{G} \mathrm{P,} \mathrm{et} \mathrm{al.} \mathrm{Trichostatin} \mathrm{A} \mathrm{improved} \mathrm{epigenetic}$ modifications of transfected cells but did not improve subsequent cloned embryo development. Anim Biotechnol, 2008, 19: 211-224

18 Whitworth K M, Zhao J, Spate L D, et al. Scriptaid corrects gene expression of a few aberrantly reprogrammed transcripts in nuclear transfer pig blastocyst stage embryos. Cell Reprogram, 2011, 13: 191-204

19 Van Thuan N, Bui H T, Kim J H, et al. The histone deacetylase inhibitor scriptaid enhances nascent mRNA production and rescues full-term development in cloned inbred mice. Reproduction, 2009, 138: $309-317$ 
20 Zhao J, Hao Y, Ross J W, et al. Histone deacetylase inhibitors improve in vitro and in vivo developmental competence of somatic cell nuclear transfer porcine embryos. Cell Reprogram, 2010, 12: $75-83$

21 Wang L J, Zhang H, Wang Y S, et al. Scriptaid improves in vitro development and nuclear reprogramming of somatic cell nuclear transfer bovine embryos. Cell Reprogram, 2011, 13: 431-439

22 Lai L, Prather R S. Production of cloned pigs by using somatic cells as donors. Cloning Stem Cells, 2003, 5: 233-241

23 Fan H Y, Sun Q Y. In vitro maturation and fertilization of pig oocytes. Methods Mol Biol, 2004, 253: 227-234

24 Tang F, Barbacioru C, Nordman E, et al. RNA-Seq analysis to capture the transcriptome landscape of a single cell. Nat Protoc, 2010, 5: 516-535

25 Santos F, Zakhartchenko V, Stojkovic M, et al. Epigenetic marking correlates with developmental potential in cloned bovine preimplantation embryos. Curr Biol, 2003, 13: 1116-1121

26 Zlatanova J, Caiafa P, Van Holde K. Linker histone binding and displacement: Versatile mechanism for transcriptional regulation. FASEB J, 2000, 14: 1697-1704

27 Hong L, Schroth G P, Matthews H R, et al. Studies of the DNA binding properties of histone $\mathrm{H} 4$ amino terminus. Thermal denaturation studies reveal that acetylation markedly reduces the binding constant of the $\mathrm{H} 4$ "tail" to DNA. J Biol Chem, 1993, 268: 305-314

28 Armstrong L, Lako M, Dean W, et al. Epigenetic modification is central to genome reprogramming in somatic cell nuclear transfer. Stem Cells, 2006, 24: 805-814

29 Cervoni N, Szyf M. Demethylase activity is directed by histone acetylation. J Biol Chem, 2001, 276: 40778-40787

30 Bui H T, Seo H J, Park M R, et al. Histone deacetylase inhibition improves activation of ribosomal RNA genes and embryonic nucleolar reprogramming in cloned mouse embryos. Biol Reprod, 2011, 85: 1048-1056

31 Costa-Borges N, Santalo J, Ibanez E. Comparison between the effects of Valproic Acid and Trichostatin A on the in vitro development, blastocyst quality, and full-term development of mouse somatic cell nuclear transfer embryos. Cell Reprogram, 2010, 12: 437-446

32 Kurosaka S, Eckardt S, McLaughlin K J. Pluripotent lineage definition in bovine embryos by Oct4 transcript localization. Biol Reprod, 2004, 71: 1578-1582

33 Niwa H, Miyazaki J, Smith A G. Quantitative expression of Oct-3/4 defines differentiation, dedifferentiation or self-renewal of ES cells. Nat Genet, 2000, 24: 372-376

34 Rodda D J, Chew J L, Lim L H, et al. Transcriptional regulation of nanog by OCT4 and SOX2. J Biol Chem, 2005, 280: 24731-24737

35 Takahashi K, Yamanaka S. Induction of pluripotent stem cells from mouse embryonic and adult fibroblast cultures by defined factors. Cell, 2006, 126: 663-676

36 du Puy L, Lopes S M, Haagsman H P, et al. Analysis of coexpression of OCT4, NANOG and SOX2 in pluripotent cells of the porcine embryo, in vivo and in vitro. Theriogenology, 2011, 75: $513-526$

37 Su J M, Wang Y S, Li Y Y, et al. Oxamflatin significantly improves nuclear reprogramming, blastocyst quality, and in vitro development of bovine SCNT embryos. PLoS One, 2011, 6: e23805

38 Cervera R P, Marti-Gutierrez N, Escorihuela E, et al. Trichostatin A affects histone acetylation and gene expression in porcine somatic cell nucleus transfer embryos. Theriogenology, 2009, 72: 1097-1110

39 Haouzi D, Hamamah S. Pertinence of apoptosis markers for the improvement of in vitro fertilization (IVF). Curr Med Chem, 2009, 16: 1905-1916

40 Meng Q, Polgar Z, Liu J, et al. Live birth of somatic cell-cloned rabbits following trichostatin A treatment and cotransfer of parthenogenetic embryos. Cloning Stem Cells, 2009, 11: 203-208

41 Martinez-Diaz M A, Che L, Albornoz M, et al. Pre- and postimplantation development of swine-cloned embryos derived from fibroblasts and bone marrow cells after inhibition of histone deacetylases. Cell Reprogram, 2010, 12: 85-94

42 Sangalli J R, De Bem T H, Perecin F, et al. Treatment of nucleardonor cells or cloned zygotes with chromatin-modifying agents increases histone acetylation but does not improve full-term development of cloned cattle. Cell Reprogram, 2012, 14: 235-247

Open Access This article is distributed under the terms of the Creative Commons Attribution License which permits any use, distribution, and reproduction in any medium, provided the original author(s) and source are credited. 